SLAC-PUB-8056

January 1999

\title{
TEST OF CPT INVARIANCE IN B FACTORIES $^{a}$
}

\author{
S. Yang \\ Physics Department, Caltech, Pasadena, CA 91125 \\ Stanford Linear Accelerator Center, Stanford University, Stanford, CA 94309
}

\begin{abstract}
Feasibility of setting limits on $C P T$ violating parameters for the case of both $C P$ conjugate and semileptonic decays of neutral $B_{d}$ mesons were examined. For the case of semileptonic final states of neutral $B_{d}$ meson decays, bounds on $C P T$ violating parameters at the level of few percent can be easily obtained. This sensitivity is better than the similar analysis using the $C P$ conjugate final states of neutral $B_{d}$ meson decays mainly due to the substantial increase on the statistics.
\end{abstract}

Invited talk presented at the Meeting on CPT and Lorentz Symmetry (CPT 98), Bloomington, IN, November 6-8, 1998.

${ }^{a}$ Work supported in part by Department of Energy grant DE-FG-03-92ER40701 and contract DE-AC03-76SF00515. 


\title{
TEST OF CPT INVARIANCE IN B FACTORIES
}

\author{
S. YANG \\ Department of Physics, 356-48 California Institute of Technology, \\ Pasadena, CA 91125, USA \\ E-mail: songhoon@SLAC.stanford.edu
}

\begin{abstract}
Feasibility of setting limits on $C P T$ violating parameters for the case of both $C P$ conjugate and semileptonic decays of neutral $B_{d}$ mesons were examined. For the case of semileptonic final states of neutral $B_{d}$ meson decays, bounds on $C P T$ violating parameters at the level of few percent can be easily obtained. This sensitivity is better than the similar analysis using the $C P$ conjugate final states of neutral $B_{d}$ meson decays mainly due to the substantial increase on the statistics.
\end{abstract}

\section{Introduction}

The $C P T$ is known to be an exact symmetry of any local relativistic quantum field theory and all known particle interactions are consistent with the hypothesis. Thus, discovery of $C P T$ violation in any form will signal new physics beyond the present particle paradigm. In fact, in a more general context, such as those arising from quantum gravity and superstring-inspired scenarios, CPT violating processes do occur, but are suppressed to levels consistent with presently existing experimental upper bounds.

At present, the most stringent bounds on $C P T$ violations are obtained from the kaon sector ${ }^{1,2}$ and are usually expressed in terms the impressively small limit on the $K^{0}-\bar{K}^{0}$ mass difference: $\left|m_{K^{0}}-m_{\bar{K}^{0}}\right| / m_{K^{0}} \leq 9 \times 10^{-19}$. Note that due to the poor knowledge we have about the possible $C P T$ violating mechanisms, there may be $C P T$ violating reactions which would be invisible to the most sensitive experiments. Therefore, though less sensitive in an absolute sense, other manifistations of $C P T$ violations, like the corresponding bounds achievable in the $D$ and $B$ sector are being investigated ${ }^{3,4}$. The realization of the proposed asymmetric $B$-factories, i.e. the PEP-II storage ring and $B A B A R$ detector at $\mathrm{SLAC}^{5}$, and the KEK-B storage ring and Belle detector at KEK ${ }^{6}$, will open a new possibility for testing $C P T$ invariance.

After the preliminary study of Kobayashi and Sanda ${ }^{7}$, the possibility of testing $C P T$ in the $B$ system has recently received a considerable attention $8,9,10$. In the present paper we will reconsider the problem, focusing on the semileptonic decays of $B_{d}$ mesons ${ }^{11}$. Similar study with $\mathrm{CP}$ conjugate final states of $B_{d}$ mesons was discussed elsewhere ${ }^{12}$. 


\section{Mixing and decay amplitudes}

The time evolution of the neutral $B$ system is described, within quantum mechanics, by a $2 \times 2$ non-hermitian Hamiltonian and the corresponding eigenvectors are given by

$$
\begin{aligned}
& \left|B_{S}\right\rangle=\frac{1}{\sqrt{2}}\left[\left(1+\epsilon_{B}+\delta_{B}\right)\left|B^{0}\right\rangle+\left(1-\epsilon_{B}-\delta_{B}\right)\left|\bar{B}^{0}\right\rangle\right], \\
& \left|B_{L}\right\rangle=\frac{1}{\sqrt{2}}\left[\left(1+\epsilon_{B}-\delta_{B}\right)\left|B^{0}\right\rangle-\left(1-\epsilon_{B}+\delta_{B}\right)\left|\bar{B}^{0}\right\rangle\right] .
\end{aligned}
$$

In the limit of exact $C P T$ symmetry $\delta_{B}=0$, whereas $\epsilon_{B}=0$ and $\delta_{B}=0$ if $C P$ is conserved. Bounds on both real and imaginary parts of $\delta_{B}$ at the level of $10 \%$ have been obtained ${ }^{3}$, and recently the imaginary part of $\delta_{B}$ has been improved to few percent level ${ }^{4}$. Note that these limits are derived under the assumption that both direct $C P T$ violating effects (i.e. $C P T$ violation in the decay amplitudes) and $\Delta B=-\Delta Q$ transitions are negligible.

We conclude this section by introducing the most general parametrization of $B^{0}$ and $\bar{B}^{0}$ semileptonic decay amplitudes.

$$
\begin{aligned}
& A^{+}=A\left(B^{0} \rightarrow l^{+} \nu h^{-}\right)=F_{h}\left(1-y_{h}\right), \\
& \bar{A}^{-}=A\left(\bar{B}^{0} \rightarrow l^{-} \nu h^{+}\right)=F_{h}^{*}\left(1+y_{h}^{*}\right), \\
& \bar{A}^{+}=A\left(\bar{B}^{0} \rightarrow l^{+} \nu h^{-}\right)=x_{h} F_{h}\left(1-y_{h}\right), \\
& A^{-}=A\left(B^{0} \rightarrow l^{-} \nu h^{+}\right)=\bar{x}_{h}^{*} F_{h}^{*}\left(1+y_{h}^{*}\right),
\end{aligned}
$$

where $h^{ \pm}$is a generic charged hadron. The exact $C P T$ symmetry implies $y_{h}=0$ and $x_{h}=\bar{x}_{h}$; the invariance under $T$ requires all the amplitudes to be real; the $\Delta B=\Delta Q$ rule implies $x_{h}=\bar{x}_{h}=0$.

\section{Time dependent distributions}

The decay amplitude to the final state $\left|a\left(\vec{q}, t_{1}\right)\right\rangle\left|b\left(-\vec{q}, t_{2}\right)\right\rangle$ is given by

$$
\begin{aligned}
A^{a, b}(t, \Delta t) \propto & \frac{1}{\sqrt{2}}\left\{A^{a} A^{b}\left(1+2 \epsilon_{B}\right) F(\Delta t)-\bar{A}^{a} \bar{A}^{b}\left(1-2 \epsilon_{B}\right) F(\Delta t)\right. \\
& \left.+\bar{A}^{a} A^{b}\left[G(\Delta t)-2 \delta_{B} F(\Delta t)\right]-A^{a} \bar{A}^{b}\left[G(\Delta t)+2 \delta_{B} F(\Delta t)\right]\right\},
\end{aligned}
$$

where

$$
t=\frac{t_{1}+t_{2}}{2}, \quad \Delta t=t_{1}-t_{2},
$$


and

$$
\begin{aligned}
& F(\Delta t)=-\sinh \left(\frac{\Delta \Gamma \Delta t}{4}\right) \cos \left(\frac{\Delta m \Delta t}{2}\right)+i \cosh \left(\frac{\Delta \Gamma \Delta t}{4}\right) \sin \left(\frac{\Delta m \Delta t}{2}\right), \\
& G(\Delta t)=+\cosh \left(\frac{\Delta \Gamma \Delta t}{4}\right) \cos \left(\frac{\Delta m \Delta t}{2}\right)-i \sinh \left(\frac{\Delta \Gamma \Delta t}{4}\right) \sin \left(\frac{\Delta m \Delta t}{2}\right) .
\end{aligned}
$$

The four terms in (3) represent the contributions due to $B^{0} B^{0}, \bar{B}^{0} \bar{B}^{0}$, $\bar{B}^{0} B^{0}$ and $B^{0} \bar{B}^{0}$ transitions to the final state. A useful observable is the socalled time difference distribution defined by

$$
I^{a b}(\Delta t)=\int_{0}^{\infty} d t_{1} d t_{2}\left|A^{a b}\left(t_{1}, t_{2}\right)\right|^{2} \delta\left(t_{1}-t_{2}-\Delta t\right)=\int_{|\Delta t|}^{\infty} d t\left|A^{a b}(t, \Delta t)\right|^{2}
$$

This quantity is proportional to $N^{a b}(\Delta t)$, i.e. to the number of events where two decay $B \rightarrow a$ and $B \rightarrow b$ occur separated by a time difference $\Delta t$ (in the $\Upsilon(4 S)$ rest frame). The time difference distributions we are interested in are those with two semileptonic decays.

Substituting in the general expression (3) the amplitudes (2) and expanding in small quantities (i.e. $\Delta B=-\Delta Q$ terms, $C P$ - and $C P T$-violating parameters, $\Delta \Gamma \Delta t$ ) up to linear terms, we obtain

$$
\begin{aligned}
I^{+-}(\Delta t)=I^{-+}(-\Delta t) & =N e^{-\Gamma|\Delta t|}\left[1+\cos (\Delta m \Delta t)-2 \Delta_{R}(\Delta \Gamma \Delta t)-4 \Delta_{I} \sin (\Delta m \Delta t)\right], \\
I^{ \pm \pm}(\Delta t) & =N e^{-\Gamma|\Delta t|}\left[1 \pm 4 \Re\left(\epsilon_{B}-y_{h}\right)\right][1-\cos (\Delta m \Delta t)],
\end{aligned}
$$

where the final states have been labeled according to the lepton sign, $N$ is a normalization factor and $\Delta_{R, I}$ are defined by

$$
\Delta_{R}=\Re \delta_{B}+\Re \frac{x_{h}-\bar{x}_{h}}{2}, \quad \Delta_{I}=\Im \delta_{B}+\Im \frac{x_{h}+\bar{x}_{h}}{2} .
$$

As can be deduced from the above formulae, the experimental study of the time difference distributions of semileptonic final states is to constraint the following three quantities: $\Delta_{R}, \Delta_{I}$ and $\Re\left(\epsilon_{B}-y_{h}\right)$.

- $\Delta_{R}$ is certainly the most interesting observable, since it is a pure index of CPT violation. Moreover, $\Re \delta_{B}$ (and thus $\Delta_{R}$ ) is the dominant $C P T$-violating term in the limit where direct $C P T$-violation is negligible. However, $\Delta_{R}$ is also the most difficult quantity to be measured, because it is multiplied by the small coefficient $\Delta \Gamma \Delta t$ in the time difference distribution (8). 
- $\Delta_{I}$ is an index of $C P T$ violation in the limit where it is possible to neglect the suppressed $C P$-violating and $\Delta B=-\Delta Q$ term $\Im\left(x_{h}+\bar{x}_{h}\right)$. Given the present bound on $C P$-violation and $\Delta B=-\Delta Q$ amplitudes in semileptonic decays, we can safely neglect the non-CPT-violating component of $\Delta_{I}$ at the level of $10^{-2}$.

A way of extracting $\Delta_{R}$ and $\Delta_{I}$ is to measure the time-dependent asymmetries which are possible to do at asymmetric $B$-factories such as $B A B A R$.

For example, the ratio of same-sign to opposite-sign di-lepton events

$$
\begin{aligned}
R(\Delta t) & =\frac{I^{++}(\Delta t)+I^{--}(\Delta t)}{I^{+-}(\Delta t)} \\
& =\frac{(1-\cos (\Delta m \Delta t))}{1+\cos (\Delta m \Delta t)-2 \Delta_{R}(\Delta \Gamma \Delta t)-4 \Delta_{I} \sin (\Delta m \Delta t)}
\end{aligned}
$$

and the asymmetry on the production time of opposite-sign di-lepton events

$$
\begin{aligned}
R^{\prime}(\Delta t) & =\frac{I^{+-}(\Delta t)-I^{-+}(\Delta t)}{I^{+-}(\Delta t)+I^{-+}(\Delta t)} \\
& =\frac{-2 \Delta_{R}(\Delta \Gamma \Delta t)-4 \Delta_{I} \sin (\Delta m \Delta t)}{1+\cos (\Delta m \Delta t)}
\end{aligned}
$$

serve the purpose. Whereas, the time-integrated asymmetry such as

$$
R=\frac{N^{++}+N^{--}}{N^{+-}}=\frac{x_{d}^{2}}{1+\left(1+8 \delta_{B}^{2}\right)\left(1+x_{d}^{2}\right)}
$$

where $x_{d}=\frac{\Delta m}{\Gamma}$ and $N^{ \pm \pm}, N^{+-}$denote number of equal and opposite-sign di-lepton events respectively, provides a bound on the magnitude of $\delta_{B}$. We derived the equation (12) under the assumption that both direct $C P T$ violating effects and $\Delta B=-\Delta Q$ transitions are negligible.

Note that the time-dependent asymmetry is linear in $\delta_{B}$ whereas the timeintegrated one is quadratic on $\delta_{B}$ hence less sensitive than the time-dependent one when the $\delta_{B}$ is small.

- $\Re\left(\epsilon_{B}-y_{h}\right)$ is presumably dominated by the $T$-violating parameter $\Re\left(\epsilon_{B}\right)$. The extraction of this quantity is the simplest one since the time dependence cancels out in the following asymmetry distribution i.e.,

$$
A_{l l}=\frac{I^{++}(\Delta t)-I^{--}(\Delta t)}{I^{++}(\Delta t)+I^{--}(\Delta t)}=4 \Re\left(\epsilon_{B}-y_{h}\right) .
$$

As noticed in reference ${ }^{3}$, an independent measurement of the direct $C P T$-violating parameter $\Re\left(y_{h}\right)$ can be in principle achieved by looking at the time-integrated inclusive asymmetries. Denoting $N_{h}^{ \pm}(>0)$ 
the total number of events where the semileptonic decay $B \rightarrow l^{ \pm} \nu h^{\mp}$ occur before any other decay (i.e. summing over all possible channels for the second vertex), one has

$$
A_{l}=\frac{N_{h}^{+}(>0)-N_{h}^{-}(>0)}{N_{h}^{+}(>0)+N_{h}^{-}(>0)}=-2 \Re y_{h} .
$$

\section{Experimental sensitivities}

A way of extracting $C P T$ violating parameters can be itemized as follows.

- Firstly, measure the time-integrated asymmetry $R$ in equation (12) to extract the magnitude of $\delta_{B}$ in the limit of both direct $C P T$ violating effects and $\Delta B=-\Delta Q$ transitions are negligible.

- Secondly, look at the time-dependent asymmetries such as $R(\Delta t)$ of equation (10) and extract $\Delta_{R}$ and $\Delta_{I}$.

- Thirdly, the above extractions on CPT violating parameters are quite sensitive to the error on asymmetry $R$. The error on asymmetry $R$ can be estimated assuming a binomial distribution for equal and oppisite-sign di-lepton events ${ }^{10}$

$$
\sigma(R)=(1+R)^{2} \sqrt{\frac{2 N^{+-} N^{++}}{N^{3}}}
$$

where, $N$ denotes total number of equal and oppisite-sign di-lepton events.

For exmaple, assuming a total luminosity of $30 \mathrm{fb}^{-1}, B_{A} B_{A R}$ expects $1.5 \times 10^{7} B^{0} \bar{B}^{0}$ pairs. With semileptonic branching fraction of $10.5 \%$ and typical lepton tagging efficiency of $50 \%$ gives about $10^{4}$ same-sign and $5 \times 10^{4}$ oppsite-sign di-lepton events. The small value of $\sigma(R)$ indicates that we can tightly constrain the $C P T$ violating parameters to few percent level.

- Lastly, we can extract the $T$-violating parameter $\Re\left(\epsilon_{B}\right)$ and the direct $C P T$-violating parameter $\Re\left(y_{h}\right)$ from the di-lepton asymmetry $A_{l l}$ of equation (13) and the inclusive single-lepton asymmetry $A_{l}$ of equation (3). If the asymmetry is small, the error on $A$ can be written as 


$$
\sigma(A) \approx \sqrt{\frac{1+\frac{B}{S}}{S}}
$$

where $S$ and $B$ denote number of signal and background events.

For exmaple, assuming $\frac{B}{S}=1$, we expect to get $\sigma(A) \approx 0.01$ with a total luminosity of $30 \mathrm{fb}^{-1}$.

\section{Conclusions}

In summary, we studied the feasibility of setting limits on $C P T$ violating parameters for the case of both $C P$ conjugate and semileptonic decays of neutral $B_{d}$ mesons. For the case of semileptonic final states of neutral $B_{d}$ meson decays, bounds on $C P T$ violating parameters at the level of few percent can be easily obtained with a total luminosity of $30 \mathrm{fb}^{-1}$. This sensitivity is better than the similar analysis using the $C P$ conjugate final states of neutral $B_{d}$ meson decays mainly due to the substantial increase on the statistics.

\section{Acknowledgments}

I thank G. Isidori for useful discussions and theoretical calculations. This work is supported in part by Department of Energy grant DE-FG-03-92ER40701 and contract DE-AC03-76SF00515.

\section{References}

1. L.K. Gibbons et al. (E731 Collaboration), Phys. Rev. D55 (1997) 6625.

2. R. Adler et al. (CPLEAR Collaboration), Phys. Lett. B364 (1995) 239.

3. D. Colladay and V.A. Kostelecký, Phys. Lett. B344 (1995) 259.

V.A. Kostelecký and R. Van Kooten, Phys. Rev. D54 (1996) 471.

4. K. Ackerstaff et al. (OPAL Collaboration), CERN-PPE/97-036 (1997).

5. BABAR Technical Design Report, SLAC-R-95-457 (1995).

6. Belle Technical Design Report, KEK-R-95-1 (1995).

7. M. Kobayashi and A.I. Sanda, Phys. Rev. Lett. 69 (1992) 3139.

8. Zhi-zhong Xing, Phys. Rev. D50 (1994) 2957.

9. A. Mohapatra et al., BELLE note 155 (1996).

10. P. Colangelo and G. Corcella, hep-ph/9704375.

11. S. Yang and G. Isidori, $B_{A} B_{A} R$ note 438 (1998).

12. S. Yang, $B_{A} B_{A R}$ note 389 (1997). 